\title{
Valve hemodynamic performance and myocardial strain after implantation of a third-generation, balloon-expandable, transcatheter aortic valve
}

\author{
Sara Fernández-Santos ${ }^{1}$, Alexis Théron ${ }^{2}$, Philippe Pibarot ${ }^{3}$, \\ Frédéric Collart ${ }^{2}$, Martine Gilard ${ }^{4}$, Marina Urena ${ }^{5}$, Tomas Hovorka ${ }^{6}$, \\ Philipp Kahlert ${ }^{7}$, José Luis Zamorano Gome ${ }^{1}$ \\ ${ }^{1}$ Cardiology Department, University Hospital Ramon y Cajal, Madrid, Spain \\ ${ }^{2}$ Department of Cardiac Surgery, La Timone Public Hospital, Marseille, France \\ ${ }^{3}$ Québec Heart and Lung Institute, Québec, Canada \\ ${ }^{4}$ Cardiology Department, La Cavalle Blanche University Hospital, Brest, France \\ ${ }^{5}$ Cardiology Department, Bichat Claude Bernard Hospital, Paris, France \\ ${ }^{6}$ Biostatistics Department, Edwards Lifesciences, Prague, Czech Republic \\ ${ }^{7}$ West German Heart and Vascular Center, Department of Cardiology and Vascular Medicine, \\ Essen University Hospital, University Duisburg-Essen, Essen, Germany
}

\begin{abstract}
Background: Left ventricular (LV) mechanics are impaired in patients with severe aortic stenosis (AS); however, transcatheter aortic valve implantation (TAVI) may positively affect LV mechanics. Assessed herein is the performance of the SAPIEN 3 transcatheter heart valve (THV) and the effect of TAVI on LV function recovery, as assessed by global longitudinal strain (GLS).

Methods: A subset of patients from the SOURCE 3 registry $(n=276)$ from 16 European centers received SAPIEN 3 balloon-expandable THV. Echocardiography was performed at baseline, postprocedure, and at 1 year, including assessment of GLS using standard two-dimensional images, and was analyzed in a core laboratory. Paired analyses between baseline and discharge, baseline and at 1 year were conducted.

Results: Hemodynamic parameters were improved after TAVI and sustained to 1 year. At 1 year, the rate of moderate to severe paravalvular leaks (PVL), and moderate to severe mitral and tricuspid regurgitations were $1.8 \%, 1.7 \%$, and $8.0 \%$, respectively. The discharge GLS $(-15.6 \pm 5.1 ; p=0.004$; $n=149)$ improved significantly from baseline $(-15.1 \pm 4.8)$ following TAVI. This improvement was sustained at 1 year compared with baseline $(-17.0 \pm 4.6, p<0.001 ; n=100)$. Conversely, LV ejection fraction (LVEF) did not significantly change following TAVI $(p=0.47)$.

Conclusions: Following TAVI with a third-generation THV, valve performances were good at 1 year with low PVL rate. The LV mechanics improved immediately after the procedure and were maintained at 1 year. These findings demonstrate the benefit of TAVI on LV mechanics, and suggests that GLS may be superior to LVEF in assessing this benefit.

Clinicaltrial.gov number: NCT02698956 (Cardiol J 2020; 27, 6: 789-796)
\end{abstract}

Key words: strain, left ventricular mechanics, echocardiography, aortic stenosis, transcatheter aortic valve implantation

Address for correspondence: Dr. Sara Fernandez Santos, Cardiology Department, University Hospital Ramon y Cajal, Carreta de Colmenar Viejo, 28034 Madrid, Spain, tel: +34 660463 557, fax: +34 913368515 ,

e-mail: sarafernandezsantos@hotmail.com

Received: 25.01.2019 Accepted: 20.04.2019 


\section{Introduction}

Degenerative aortic stenosis (AS) is one of the most prevalent cardiovascular diseases in developed countries. Over the past decade, transcatheter aortic valve implantation (TAVI) has emerged as the therapy of choice for patients with AS considered inoperable or at high surgical risk $[1,2]$. TAVI has improved the prognosis of these patients. And, as transcatheter heart valves (THV) have evolved, patients who had received TAVI suffered fewer complications.

Aortic stenosis induces a series of adaptive responses. It generates a pressure overload that alters left ventricular (LV) geometry and performance; although, LV volume and LV ejection fraction (LVEF) may be preserved, even in advanced stages of the disease [3]. The most important changes caused by the pressure overload include hypertrophic remodeling [4], diastolic dysfunction [5, 6], and impaired contractility [7].

Patients with AS who are treated with TAVI can experience relief from this pressure overload that is reflected in changes in LV strain [4]. The immediate result of TAVI is often an acute decrease in transvalvular gradient, leading to an improvement of LV mechanics. This could be a precursor to, or a reverse in, remodeling, possibly leading to a reduction in LV mass and an improvement in long-term diastolic function.

Studies have demonstrated that strain (global longitudinal strain [GLS]) imaging is the most appropriate method to evaluate subtle changes in myocardial function that occur in patients with AS $[8,9]$. Additionally, GLS is independently predictive of mortality [10]. In a recently published study of 92 patients treated in Europe with either the self-expanding CoreValve (Medtronic, Minneapolis, MN, USA) or the mechanically expanded Lotus valve (Boston Scientific, Natick, MA, USA), TAVI was associated with an immediate improvement in LV mechanics, as demonstrated by GLS increase; although LV systolic function remained unaltered [4].

The literature on post implantation LV mechanics is limited. The impact of TAVI on LV mechanics using GLS in patients who received the SAPIEN 3 (Edwards Lifesciences; Irvine, CA, USA) balloon-expandable, transcatheter valve at 1 year follow-up were analyzed.

\section{Methods}

\section{Study population}

Patients with symptomatic, severe AS were implanted with the third-generation, balloonexpandable SAPIEN THV (SAPIEN 3). The selection of patients was based on a clinical consensus of the Heart Team. A subset of patients from the SOURCE 3 registry had planned, per protocol, to have their echocardiograms reviewed by an independent central echocardiography core laboratory (ECL; Ramon y Cajal, Madrid). Patients had echocardiograms at baseline, discharge, and at 1 year after implantation.

\section{Intervention and purpose}

The SAPIEN 3 Aortic Bioprosthesis European Outcome (SOURCE 3) is a European, post-approval multicenter, observational registry, aimed to evaluate the safety and performance of the SAPIEN 3 THV under real-world conditions. The full cohort of 30-day and 1-year results had been published previously [11, 12]. A protocol was developed for this echocardiographic sub-study. It was approved by the local ethics committees and the respective health authorities in participating countries (France, Germany, United Kingdom, and Italy). All patients provided written, informed consent before the study commenced.

Clinical outcomes (cardiac death and disabling stroke to 1 year and life-threatening bleedings to 30 days) were adjudicated by a clinical event committee.

Patients had two-dimensional (2D) transthoracic echocardiograms according to the protocol. The sites sent the echocardiograms to the core laboratory for comprehensive evaluation of hemodynamic performance of valve and LV function. The protocol stipulated multiple echocardiographic measurements before and after prosthesis implantation, as well as quantification of LV mechanics, measuring LV strain with standard 2D imaging (Image Arena and CPA package, TomTec Imaging System). The assessment of GLS was done using averages of measures taken from images on three views: apical 4-, 3- and 2-chamber views in an 18-segment LV model. To obtain LV strain measurements, endocardial contour needed to be manually outlined, after which the system generated the myocardial perimeter on the end systolic 
Table 1. Baseline characteristics of the SOURCE 3 cohort.

\begin{tabular}{|c|c|c|c|}
\hline & $\begin{array}{l}\text { Patients with AS } \\
\text { who received } \\
\text { SAPIEN } 3 \text { THV } \\
\text { (n=276) }\end{array}$ & $\begin{array}{l}\text { Patients with AS who } \\
\text { received SAPIEN } 3 \text { THV, } \\
\text { with no ECL assessment } \\
\text { (n=1670) }\end{array}$ & $\mathbf{P}$ \\
\hline \multicolumn{4}{|l|}{ Demographics and clinical variables } \\
\hline Age [years], mean \pm SD & $80.8 \pm 7.47$ & $81.7 \pm 6.49$ & 0.124 \\
\hline Age $\geq 80$ years & $184(66.7 \%)$ & $1136(68.0 \%)$ & 0.677 \\
\hline Female & $126(45.7 \%)$ & $809(48.4 \%)$ & 0.399 \\
\hline Logistic EuroScore, mean \pm SD & $15.6 \pm 10.60, N=226$ & $18.7 \pm 13.46, N=1558$ & 0.002 \\
\hline EuroScore II, mean \pm SD & $4.6 \pm 3.98, N=204$ & $5.7 \pm 5.71, N=1295$ & 0.007 \\
\hline NYHA class IV & $19(7.0 \%), N=272$ & $150(9.3 \%), N=1607$ & $<0.001$ \\
\hline Hypertension & $199(72.1 \%)$ & $1392(83.4 \%)$ & 0.090 \\
\hline Dyslipidaemia & $136(49.3 \%)$ & $918(55.0 \%)$ & 0.103 \\
\hline History of smoking & $95(34.4 \%)$ & $490(29.4 \%), N=1669$ & 0.117 \\
\hline Diabetes & $70(25.4 \%)$ & $504(30.2 \%)$ & 0.194 \\
\hline Coronary artery disease & $132(47.8 \%)$ & $870(52.1 \%)$ & 0.546 \\
\hline Myocardial infarction & $29(10.5 \%)$ & $199(11.9 \%)$ & 0.414 \\
\hline Coronary bypass grafting & $27(9.8 \%)$ & $194(11.6 \%), N=1669$ & 0.001 \\
\hline Congestive heart failure & $129(46.7 \%)$ & $577(34.6 \%)$ & 0.561 \\
\hline Renal insufficiency & $80(29.0 \%)$ & $455(27.2 \%)$ & 0.039 \\
\hline Percutaneous coronary intervention & $78(28.3 \%)$ & $580(34.8 \%), N=1669$ & 0.124 \\
\hline \multicolumn{4}{|l|}{ Aortic valve severity } \\
\hline Mitral regurgitation (degree moderate to severe) & $24(9.2), N=260$ & $224(14.8), N=1513$ & 0.015 \\
\hline Tricuspid regurgitation (moderate to severe) & $18(7.3), N=245$ & $162(11.5), N=1404$ & 0.059 \\
\hline
\end{tabular}

$P$ values are from the Wilcoxon sum rank test for the continuous variables and the Fisher exact test for categorical variables. AS - aortic stenosis; ECL — echocardiology core laboratory; LV — left ventricle; NYHA — New York Heart Association; SD — standard deviation; THV - transcatheter heart valve

frame. Images of measures in a patient with baseline, discharge, and 1-year measures are displayed in supplementary files (Suppl. Images 1 and 2).

Two experienced cardiologists examined all echocardiographic data. Intraoperatory aortograms were also performed during valve implantation by many participating sites. A hemodynamic cardiologist from the core laboratory, who was blinded to the echocardiographic results, evaluated these studies.

The aim of the study was to evaluate the impact of TAVI on myocardial longitudinal LV systolic strain in patients with severe, degenerative AS. Additionally, the ECL evaluated hemodynamic parameters.

\section{Statistical analysis}

Study staff at participating centers entered echocardiographic data into an electronic capture system. The Sponsor monitored it before it was sent to the ECL. Comparisons of baseline and procedural characteristics between the subset of patients analyzed and the rest of the SOURCE 3 cohort were conducted using the Wilcoxon sum rank test for the continuous variables and the Fisher exact test for categorical variables.

Echocardiographic parameters were compared between discharge and at 1 year, using paired analysis with the Wilcoxon sum rank test. Mean gradient, effective orifice area (EOA), and GLS were compared (baseline vs. discharge and baseline vs. 1 year), using a paired analysis with the t test.

\section{Results}

\section{Baseline and procedural data}

A total of 276 patients were enrolled in the echocardiographic analysis between July 2014 and October 2015 in 16 European centers. In summary, patient baseline characteristics were a mean age of 80.8 years and a mean EuroSCORE II of $4.6 \pm 3.98$ (Table 1). The latter was statistically lower than the mean EuroSCORE II of the SOURCE 3 patients not included in this sub-analysis $(\mathrm{p}=0.002)$, as more echo patients had a logistic EuroSCORE of $<10 \%$, 
compared with other patients of the SOURCE 3 cohort (35.0\% vs. $28.2 \%$; $\mathrm{p}=0.041)$, and fewer echo patients had a logistic EuroSCORE of $>30 \%$ compared with other patients of the cohort $(10.2 \%$ vs. $16.6 \% ; \mathrm{p}=0.011)$. Most other baseline clinical characteristics and comorbidities were comparable between the subset of patients analyzed and the rest of the SOURCE 3 cohort, except for hypertension $(72.1 \%$ in the echo patients vs. $83.4 \%$ in other SOURCE 3 patients; $\mathrm{p}<0.001)$, congestive heart failure (46.7\% vs. $34.6 \%$; $<<0.001$ ), and mitral regurgitation of moderate or severe grade $(9.2 \%$ vs. $14.8 \%$; $\mathrm{p}=0.015)$.

Most TAVI procedures were performed using a transfemoral approach $(87.3 \%)$, with the SAPIEN 3 THV $23 \mathrm{~mm}$ (40.6\%), $26 \mathrm{~mm}$ (36.2\%), and $29 \mathrm{~mm}(23.2 \%)$.

An intraprocedural angiography was retrieved in 103 patients; most were adjudicated as grade 1 , but a small percentage were considered grade 2 (Table 2 ).

In terms of clinical outcomes, the cohort had a 30 -day and 1-year mortality rate of $1.5 \%$ and $5.4 \%$, respectively. The cardiac mortality rate was $0.7 \%$ and $3.1 \%$, at 30 days and 1 year, respectively. The disabling stroke rate was $0.7 \%$ and $1.1 \%$, at 30 days and 1 year, respectively. The life-threatening bleeding rate was $5.4 \%$ at 30 days.

\section{Echocardiographic parameters}

Aortic regurgitation severity was predominantly grade 1 , using both the Seller and Valve Academic Research Consortium-2 criteria (93.2\% each; Table 3). Other echocardiographic parameters are presented in Table 4.

\section{Effective orifice area and mean gradient}

The TAVI treatment significantly improved the mean EOA from $0.8 \pm 0.3 \mathrm{~cm}^{2}$ at baseline to $1.6 \pm 0.6 \mathrm{~cm}^{2}$ at discharge (Fig. 1, Table 4). This improvement was sustained at 1 year $\left(1.5 \pm 0.5 \mathrm{~cm}^{2}\right.$; $\mathrm{p}<0.001$ compared with baseline). Similarly, the mean gradient was decreased following the THV treatment from $41.2 \pm 14.6 \mathrm{mmHg}$ at baseline to $12.2 \pm 5.3 \mathrm{mmHg}$ at discharge $(\mathrm{p}<0.001)$, and was maintained at 1 year $(12.7 \pm 5.8 \mathrm{mmHg}$; $\mathrm{p}<0.001$ compared with baseline)

\section{Total aortic regurgitation and PVL}

Few patients had total aortic regurgitation (TAR) at discharge; it was moderate severity in $5(2.1 \%)$ patients and severe in $2(0.8 \%)$ patients (Fig. 2). At 1 year, 3 (1.7\%) patients had moderate TAR; no severe TAR was observed (Fig. 2).
Table 2. Procedural characteristics.

\begin{tabular}{lc}
\hline $\begin{array}{l}\text { Procedural } \\
\text { characteristics }\end{array}$ & $\begin{array}{c}\text { Patients who } \\
\text { received } \\
\text { echocardiograms } \\
\text { (n = 276) }\end{array}$ \\
\hline Total procedure time [min] & $\begin{array}{c}72.1 \pm 52.23 \\
(\mathrm{n}=204)\end{array}$ \\
Total anaesthesia time [min] & $\begin{array}{c}127.0 \pm 84.71 \\
(\mathrm{n}=127)\end{array}$ \\
Access approach: & \\
Transfemoral & $241(87.3 \%)$ \\
Transapical & $26(9.4 \%)$ \\
Transaortic & $7(2.5 \%)$ \\
Implanted valve size [mm]: & $112(40.6 \%)$ \\
$23 \mathrm{~mm}$ & $100(36.2 \%)$ \\
$26 \mathrm{~mm}$ & $64(23.2 \%)$ \\
$29 \mathrm{~mm}$ &
\end{tabular}

Table 3. Aortic regurgitation severity.

\begin{tabular}{cc}
\hline Criteria & $\begin{array}{c}\text { Patients } \\
\text { who received } \\
\text { intraprocedural } \\
\text { angiography } \\
\text { (n = 103) }\end{array}$ \\
\hline Evaluation with Seller's criteria: & $96(93.2 \%)$ \\
Grade 1 & $7(6.8 \%)$ \\
Grade 2 & $0(0 \%)$ \\
Grade 3 & $0(0 \%)$ \\
Grade 4 & $96(93.2 \%)$ \\
Evaluation with VARC 2 criteria: & $7(6.8 \%)$ \\
Grade 1 & $0(0 \%)$ \\
Grade 2 & $0(0 \%)$ \\
Grade 3 & \\
Grade 4 &
\end{tabular}

VARC - Valve Academic Research Consortium

Similarly, few severe to moderate paravalvular leak (PVLs) were present at discharge (2.9\%) and 1 year (1.8\%; Fig. 2).

\section{Mitral and tricuspid regurgitation}

At discharge, 2 patients had moderate severity mitral regurgitation and 2 had severe mitral regurgitation (Fig. 3). At 1 year, 3 patients had moderate mitral regurgitation. The percentage of mild mitral regurgitation was significantly lower at 1 year compared with discharge (20.3\% vs. $28.3 \%$, respectively; $p=0.011$ ). 
Table 4. Echocardiographic parameters.

\begin{tabular}{|c|c|c|c|c|c|}
\hline Parameter & $\begin{array}{c}\text { Baseline } \\
\text { Mean } \pm \text { SD } \\
(\mathrm{n})\end{array}$ & $\begin{array}{c}\text { Discharge } \\
\text { Mean } \pm \text { SD } \\
(\mathrm{n})\end{array}$ & $\begin{array}{c}1 \text { year } \\
\text { Mean } \pm \text { SD } \\
(\mathrm{n})\end{array}$ & $\begin{array}{c}P^{*}(\mathbf{n}) \\
\text { Baseline } \\
\text { vs. discharge }\end{array}$ & $\begin{array}{l}\quad P^{*}(n) \\
\text { Discharge } \\
\text { vs. } 1 \text { year }\end{array}$ \\
\hline LVEDV [mL] & $81.3 \pm 36.0(211)$ & $76.0 \pm 36.1(183)$ & $80.0 \pm 33.7(130)$ & $0.013(151)$ & $0.316(85)$ \\
\hline LVESV [mL] & $36.3 \pm 26.5(211)$ & $34.8 \pm 26.0(183)$ & $35.5 \pm 26.2(129)$ & $0.028(151)$ & $0.760(85)$ \\
\hline LVEDD [cm] & $4.8 \pm 0.8(221)$ & $4.7 \pm 0.8(187)$ & $4.7 \pm 0.8(123)$ & $0.109(162)$ & $0.160(92)$ \\
\hline LVESD [cm] & $3.2 \pm 1.0(210)$ & $3.2 \pm 1.00(183)$ & $3.1 \pm 0.9(119)$ & $0.036(152)$ & $0.325(88)$ \\
\hline LVEF [\%] & $58.6 \pm 15.6(211)$ & $57.5 \pm 14.9(183)$ & $58.8 \pm 13.2(129)$ & $0.471(151)$ & $0.712(85)$ \\
\hline $\begin{array}{l}\text { LV posterior wall } \\
\text { diastolic }\end{array}$ & $1.1 \pm 0.2(219)$ & $1.2 \pm 0.2(187)$ & $1.1 \pm 0.2(120)$ & $0.123(162)$ & $0.025(90)$ \\
\hline $\begin{array}{l}\text { Interventricular septum } \\
\text { diastolic }\end{array}$ & $1.3 \pm 0.3(221)$ & $1.4 \pm 0.3(192)$ & $1.3 \pm 0.2(120)$ & $0.235(166)$ & $0.210(93)$ \\
\hline Left atrial volume [mL] & $75.6 \pm 35.5(237)$ & $77.4 \pm 32.2(212)$ & $74.9 \pm 29.6(163)$ & $0.754(186)$ & $0.380(124)$ \\
\hline $\begin{array}{l}\text { AV mean gradient } \\
{[\mathrm{mmHg}]}\end{array}$ & $\begin{array}{c}41.2 \pm 14.6 \\
(251)\end{array}$ & $\begin{array}{l}12.2 \pm 5.3 \\
\quad(242)\end{array}$ & $\begin{array}{l}12.7 \pm 5.8 \\
\quad(178)\end{array}$ & $\begin{array}{c}<0.001 \\
(223)\end{array}$ & $\begin{array}{l}0.025 \\
(155)\end{array}$ \\
\hline$A V$ area $(E O A)\left[\mathrm{cm}^{2}\right]$ & $0.8 \pm 0.3(227)$ & $1.6 \pm 0.6(202)$ & $1.5 \pm 0.5(159)$ & $<0.001(173)$ & $0.007(120)$ \\
\hline $\begin{array}{l}\text { AV velocity time } \\
\text { integral }\end{array}$ & $\begin{array}{c}96.7 \pm 22.6 \\
(251)\end{array}$ & $\begin{array}{c}44.0 \pm 11.4 \\
(240)\end{array}$ & $\begin{array}{c}49.6 \pm 13.8 \\
(178)\end{array}$ & $\begin{array}{c}<0.001 \\
(221)\end{array}$ & $\begin{array}{c}<0.001 \\
(153)\end{array}$ \\
\hline $\begin{array}{l}\text { Mitral annulus } \\
\text { velocity }[\mathrm{cm} / \mathrm{s}]\end{array}$ & $6.1 \pm 1.9(99)$ & $6.2 \pm 2.0(89)$ & $6.5 \pm 2.4(85)$ & 0.911 (47) & $0.167(44)$ \\
\hline $\begin{array}{l}\text { E/e' ratio (filling } \\
\text { pressures) }[\mathrm{mmHg}]\end{array}$ & $17.9 \pm 7.9(96)$ & $17.2 \pm 8.1(86)$ & $17.8 \pm 8.1(80)$ & $0.920(44)$ & $0.917(41)$ \\
\hline $\begin{array}{l}\text { Systolic pulmonary } \\
\text { pressure }[\mathrm{mmHg}]\end{array}$ & $\begin{array}{c}302.2 \pm 52.0 \\
(47)\end{array}$ & $\begin{array}{c}273.2 \pm 42.25 \\
(59)\end{array}$ & $\begin{array}{c}282.2 \pm 45.8 \\
(65)\end{array}$ & $\begin{array}{c}0.843 \\
(14)\end{array}$ & - \\
\hline
\end{tabular}

*P values are from the Wilcoxon sum rank (paired) test. Mean \pm standard deviation (SD) presented in the 3 first columns were calculated on all values available. AV — atrio-ventricular; EOA - effective orifice area; LVEDD — left ventricular end diastolic diameter; LVEF — left ventricular ejection fraction; LVEDV - left ventricular end-diastolic volume; LVESD - left ventricular end-systolic diameter; LVESV — left ventricular end-systolic volume

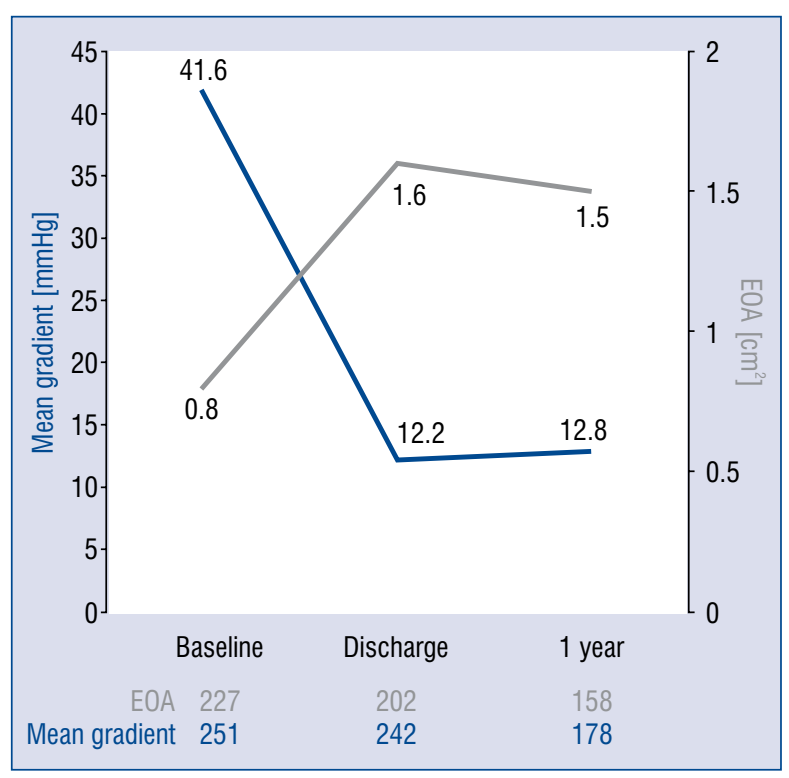

Figure 1. Effective orifice area and mean gradient paired analyses.

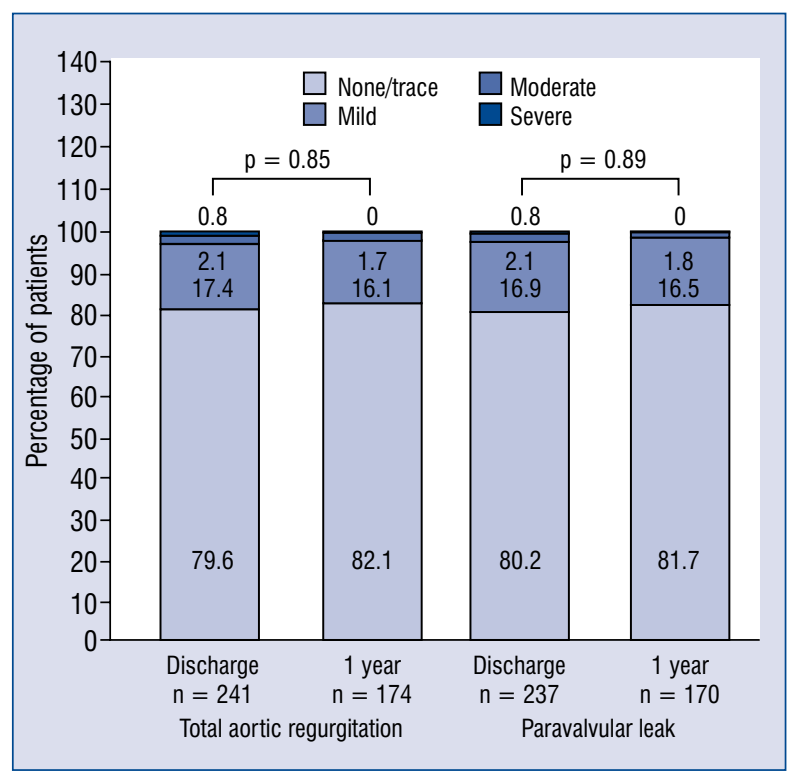

Figure 2. Total aortic regurgitation and paravalvular leak - paired analyses. 


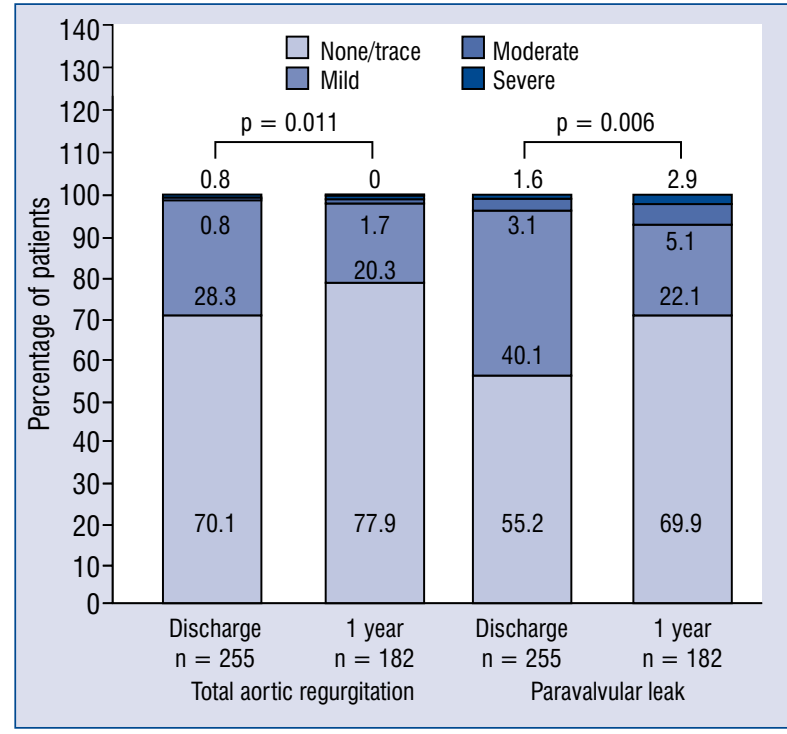

Figure 3. Mitral regurgitation and tricuspid regurgitation - paired analyses.

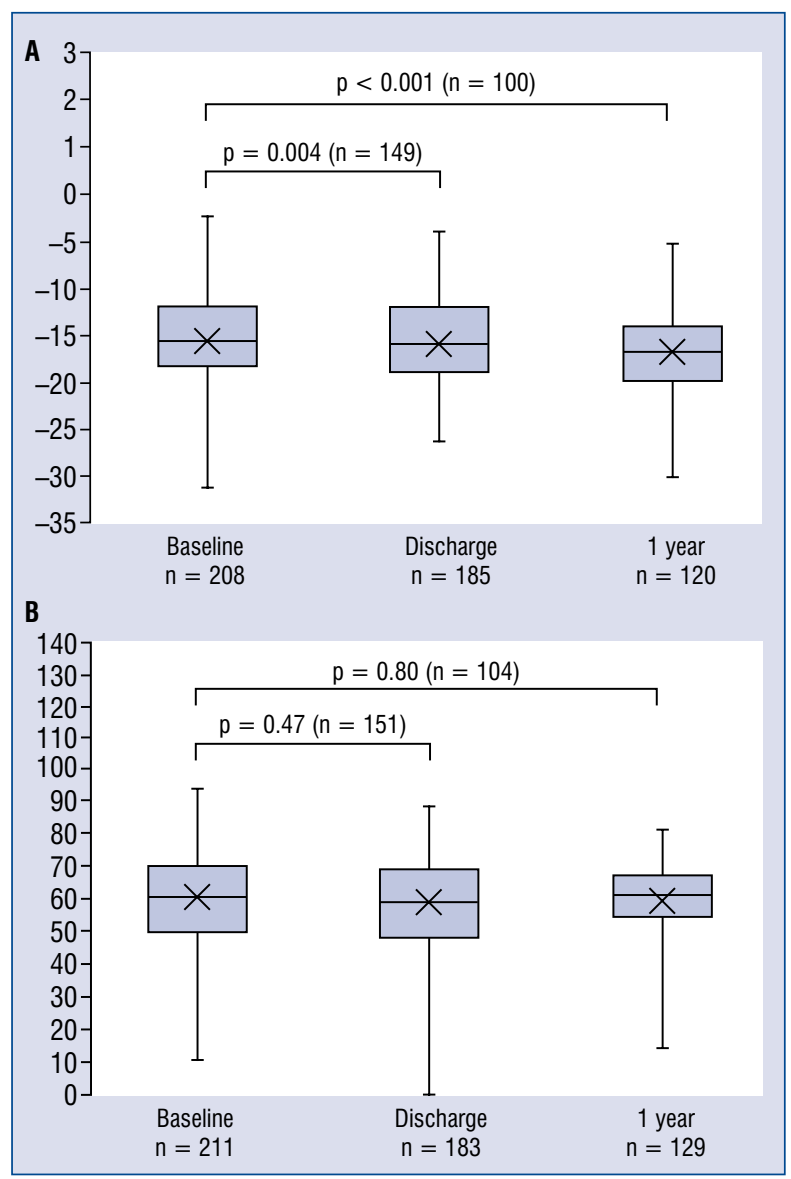

Figure 4. A. Global longitudinal strain analysis; B. Left ventricular ejection fraction; the box plot represents the mean \pm standard deviation, median, minimum and maximum (whiskers) of the global longitudinal strain. $P$ values compare baseline with discharge, and baseline with 1-year data using the paired t-test.
Similarly, 6 patients suffered from tricuspid regurgitation of moderate severity at discharge and 3 others presented with severe tricuspid regurgitation (Fig. 3). At 1 year, 7 patients presented with moderate tricuspid regurgitation and 4 patients with severe tricuspid regurgitation. The percentage of mild mitral tricuspid regurgitation was significantly lower at 1 year compared with discharge ( $22.1 \%$ vs. $40.1 \%$, respectively; $\mathrm{p}=0.006)$.

\section{Global longitudinal strain analysis}

The LV peak systolic longitudinal strain significantly improved after TAVI (Fig. 4A), and significantly increased at 1 year compared with baseline $(-17.0 \pm 4.6 ; \mathrm{p}=0.001)$. No change was observed on the LVEF (Fig. 4B).

\section{Discussion}

This echocardiographic evaluation performed in a real-world setting in European patients with severe AS who received a transcatheter SAPIEN 3 demonstrated good valve performance, low PVL of moderate to severe grade at 1 year, and statistically significant improvement in LV function as assessed by GLS. No change in LVEF was observed.

\section{Population studied}

It was thought that the population analyzed in the present study was representative of patients with severe AS and were usually referred for the TAVI procedure. Demographic and clinical parameters at baseline were comparable with those of the entire SOURCE 3 cohort, except for a slightly lower surgical risk score in the SOURCE 3 cohort.

\section{Echocardiographic parameters}

A comprehensive echocardiographic assessment from randomized trials, including the PARTNER 2 SAPIEN 3 registry, presented comparable mean gradient and EOA at discharge or at 30 days (mean gradient of $11.18 \pm 4.35 \mathrm{mmHg}$ and EOA of $\left.1.66 \pm 0.38 \mathrm{~cm}^{2} ; \mathrm{n}=1470\right)$ as assessed by the ECL [13].

One potential disadvantage of TAVI is an increased incidence of post-procedural aortic regurgitation, which is an independent predictor of short- and long-term mortality, and which may have a negative impact on LV myocardial recovery [14, 15]. The presence of post-procedural PVL appears to limit LV structural and functional recovery [16]. Post-procedural PVL was rare at 1 year and no patients presented with severe PVL. 


\section{Left ventricular strain analysis}

The first signs of reverse LV remodeling at discharge were observed, and were sustained for 1 year. A significant increase in GLS was numerically modest, but statistically significant, observed at discharge and not only sustained, but also improved at 1 year. This result represents signs of reverse remodeling, as previously reported in TAVI $[4,17]$ or surgical aortic valve replacement studies [7]. Several studies have demonstrated an amelioration in LV mass [18], some diastolic filling parameters $[18,19]$, and left atrial function in patients after TAVI $[16,19]$. The LVEF is confounded by the positive remodeling of the left ventricle, i.e., regression of $L V$ concentric hypertrophy; LVEF is not a good marker of LV intrinsic myocardial function.

\section{Limitations of the study}

A few patients were not evaluable, mainly because their echocardiographs were of poor quality, so there may have been selection bias.

One of the limitations of the study is loss to follow-up of some patients. The reason for this was due to the multicenter recruitment: many patients travelled far to have the TAVI procedure and could not return for the 12-month echo. However, updates were received from the sites and local phone calls related to the absence of mortality in non-returning patients. In addition, some studies were excluded from analysis due to the poor quality of examinations.

This SOURCE 3 sub-study was designed as a purely echocardiographic study. Consequently, no clinical parameters were collected in follow-up, including those affecting quality of life data. Further studies are required to seek correlation between echocardiographic improvement in LV mechanics and clinical response.

\section{Conclusions}

A subset of patients from the SOURCE 3 registry who received the SAPIEN 3 balloon-expandable THV had improved LV mechanics immediately following the procedure that were sustained for 1 year, as determined by standard 2D imaging. The valve performance was good at 1 year, with a low PVL rate.

\section{Acknowledgements}

Frederique Maneval of Edwards Lifesciences provided medical writing services, and Tracey Fine of Edwards Lifesciences provided medical editing services.
Funding: Edwards Lifesciences funded this study.

Conflict of interest: Philipp Kahlert has received honoraria as a clinical proctor for Edwards Lifesciences Inc. Tomas Hovorka is an employee of Edwards Lifesciences. Other authors did not declare any conflicts.

\section{References}

1. Leon MB, Smith CR, Mack M, et al. PARTNER Trial Investigators. Transcatheter aortic-valve implantation for aortic stenosis in patients who cannot undergo surgery. N Engl J Med. 2010; 363(17): 1597-1607, doi: 10.1056/NEJMoa1008232, indexed in Pubmed: 20961243.

2. Smith C, Leon M, Mack M, et al. Transcatheter versus Surgical Aortic-Valve Replacement in High-Risk Patients. N Engl J Med. 2011; 364(23): 2187-2198, doi: 10.1056/nejmoa1103510.

3. Ross J. Afterload mismatch and preload reserve: a conceptual framework for the analysis of ventricular function. Prog Cardiovasc Dis. 1976; 18(4): 255-264, indexed in Pubmed: 128034.

4. Lozano Granero VC, Fernández Santos S, Fernández-Golfín C, et al. Immediate improvement of left ventricular mechanics following transcatheter aortic valve replacement. Cardiol J. 2018; 25(4): 487-494, doi: 10.5603/CJ.a2018.0066, indexed in Pubmed: 29924376.

5. Chang SA, Park PW, Sung K, et al. Noninvasive estimate of left ventricular filling pressure correlated with early and midterm postoperative cardiovascular events after isolated aortic valve replacement in patients with severe aortic stenosis. J Thorac Cardiovasc Surg. 2010; 140(6): 1361-1366, doi: 10.1016/j. jtcvs.2010.02.022, indexed in Pubmed: 20381086.

6. Dahl JS, Videbæk L, Poulsen MK, et al. Noninvasive assessment of filling pressure and left atrial pressure overload in severe aortic valve stenosis: relation to ventricular remodeling and clinical outcome after aortic valve replacement. J Thorac Cardiovasc Surg. 2011; 142(3): e77-e83, doi: 10.1016/j.jtcvs.2011.01.032, indexed in Pubmed: 21353251.

7. Delgado V, Tops LF, van Bommel RJ, et al. Strain analysis in patients with severe aortic stenosis and preserved left ventricular ejection fraction undergoing surgical valve replacement. Eur Heart J. 2009; 30(24): 3037-3047, doi: 10.1093/eurheartj/ehp351, indexed in Pubmed: 19726436.

8. Weidemann F, Jamal F, Sutherland GR, et al. Myocardial function defined by strain rate and strain during alterations in inotropic states and heart rate. Am J Physiol Heart Circ Physiol. 2002; 283(2): H792-H799, doi: 10.1152/ajpheart.00025.2002, indexed in Pubmed: 12124229.

9. Weidemann F, Jamal F, Kowalski M, et al. Can strain rate and strain quantify changes in regional systolic function during dobutamine infusion, B-blockade, and atrial pacing--implications for quantitative stress echocardiography. J Am Soc Echocardiogr. 2002; 15(5): 416-424, indexed in Pubmed: 12019424.

10. Kusunose K, Goodman A, Parikh R, et al. Incremental prognostic value of left ventricular global longitudinal strain in patients with aortic stenosis and preserved ejection fraction. Circ: Cardiovasc Imaging. 2014; 7(6): 938-945, doi: 10.1161/circimaging.114.002041. 
11. Wendler O, Schymik G, Treede H, et al. SOURCE 3 Registry: Design and 30-Day Results of the European Postapproval Registry of the Latest Generation of the SAPIEN 3 Transcatheter Heart Valve. Circulation. 2017; 135(12): 1123-1132, doi: 10.1161/CIRCULATIONAHA.116.025103, indexed in Pubmed: 28104716.

12. Wendler O, Schymik G, Treede H, et al. SOURCE 3: 1-year outcomes post-transcatheter aortic valve implantation using the latest generation of the balloon-expandable transcatheter heart valve. Eur Heart J. 2017; 38(36): 2717-2726, doi: 10.1093/eurheartj/ehx294, indexed in Pubmed: 28605423.

13. Hahn RT, Leipsic J, Douglas PS, et al. Comprehensive echocardiographic assessment of normal transcatheter valve function. JACC Cardiovasc Imaging. 2019; 12(1): 25-34, doi: 10.1016/j. jcmg.2018.04.010, indexed in Pubmed: 29909110.

14. Généreux P, Head SJ, Hahn R, et al. Paravalvular leak after transcatheter aortic valve replacement: the new Achilles' heel? A comprehensive review of the literature. J Am Coll Cardiol. 2013; 61(11): 1125-1136, doi: 10.1016/j.jacc.2012.08.1039, indexed in Pubmed: 23375925.

15. Lerakis S, Hayek SS, Douglas PS. Paravalvular aortic leak after transcatheter aortic valve replacement: current knowledge.
Circulation. 2013; 127(3): 397-407, doi: 10.1161/CIRCULATIONAHA.112.142000, indexed in Pubmed: 23339094.

16. Poulin F, Carasso S, Horlick EM, et al. Recovery of left ventricular mechanics after transcatheter aortic valve implantation: effects of baseline ventricular function and postprocedural aortic regurgitation. J Am Soc Echocardiogr. 2014; 27(11): 1133-1142, doi: 10.1016/j.echo.2014.07.001, indexed in Pubmed: 25125314.

17. D'Andrea A, Padalino R, Cocchia R, et al. Effects of transcatheter aortic valve implantation on left ventricular and left atrial morphology and function. Echocardiography. 2015; 32(6): 928-936, doi: 10.1111/echo.12808, indexed in Pubmed: 25323699.

18. Vizzardi E, D'Aloia A, Fiorina C, et al. Early regression of left ventricular mass associated with diastolic improvement after transcatheter aortic valve implantation. J Am Soc Echocardiogr. 2012; 25(10): 1091-1098, doi: 10.1016/j.echo.2012.06.010, indexed in Pubmed: 22819229.

19. Spethmann S, Dreger H, Baldenhofer G, et al. Short-term effects of transcatheter aortic valve implantation on left atrial mechanics and left ventricular diastolic function. J Am Soc Echocardiogr. 2013; 26(1): 64-71.e2, doi: 10.1016/j.echo.2012.10.002, indexed in Pubmed: 23140843. 